\title{
Getting to the heart of NAFLD
}

New research demonstrating marked changes in cardiac structure and function in adults with NAFLD confirms that the effects of NAFLD extend beyond the liver.

"NAFLD is associated with an excess cardiovascular disease risk, which may represent a greater burden of the condition at a population level," explains author Michael Trenell from Newcastle University, UK. "Despite the increased likelihood of cardiovascular complications, surprisingly little is known about cardiac function in people with NAFLD," he adds.

As such, the authors wanted to better define cardiac structure, function and metabolism in the context of NAFLD. To do so, they enrolled to their study 19 adults with NAFLD and 19 healthy individuals without liver or metabolic disease as controls (matched by age, sex and BMI, among others).

Next, they examined cardiac structure (such as wall thickness), function (contractile properties) and phosphate metabolism using highly sensitive magnetic-resonance-based techniquesincluding MRI, cardiac tagging and magnetic resonance spectroscopy-in a bid to detect potentially subclinical changes to cardiac health.

"The results show, for the first time, subtle exaggerations in both ventricular wall thickness and movement during each heartbeat in people with NAFLD," states Trenell. Individuals with NAFLD had markedly thicker left ventricular walls at systole (14 \pm 3 versus $12 \pm 2 \mathrm{~mm}, P<0.01$ ) and diastole $(8 \pm 1$ versus $7 \pm 1 \mathrm{~mm}$, $P<0.01)$ than controls, independent of left ventricular mass. Individuals with fatty liver also had decreased longitudinal shortening.

Altered myocardial strains as well as evidence of concentric remodelling (that is, changes to the physiology and pathological state of the heart, in this case thickened walls relative to the whole size of the heart) were also observed in patients with NAFLD, but not in controls.

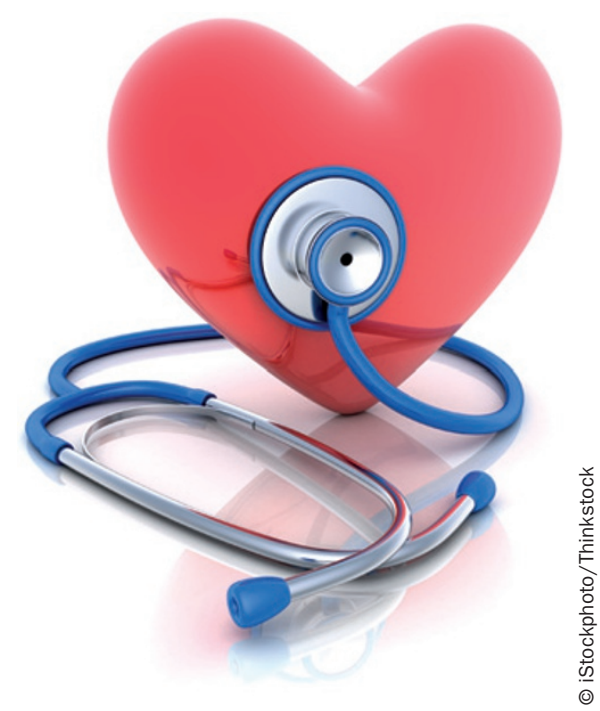

No differences in cardiac metabolism or energetics were observed between the two study groups. Moreover, all changes in cardiac function and structure were observed in the absence of overt cardiac disease.

"One of the most encouraging observations in this study is that the changes are subtle and subclinical in their severity," says Trenell. Such changes would be a useful target for therapy to minimise the risk of progression to cardiovascular disease at an early stage. Trenell notes: "the findings also suggest that effectively managing NAFLD confers benefit beyond liver fat and metabolic control and should not be underestimated".

The investigators now hope to expand on previous work by exploring how liver fat and cardiac function could be modified using lifestyle therapy (such as exercise and weight loss), which are interventions that have already proved useful for the treatment of NAFLD itself.

\section{Katrina Ray}

Original article Hallsworth, K. et al. Cardiac structure and function are altered in adults with non-alcoholic fatty liver disease. J. Hepatol. doi:10.1016/j.jhep.2012.11.015 